\title{
NOVOS SURFACTANTES ALQUIL POLIGLICOSÍDICOS À BASE DE AMILOSE EXTRAÍDA DA BATATA INGLESA (Solanum tuberosum L.)
}

\author{
Francisco C. F. de França ${ }^{\mathrm{a}, \mathrm{b}}$, Denise R. Moreira ${ }^{\mathrm{b}}$, Raimundo R. de Almeida ${ }^{\mathrm{b}}$, Francisco H. A. Rodrigues ${ }^{\mathrm{c}}$, Maria E. N. P. \\ Ribeiro $^{\mathrm{b}, *}$ e Nágila M. P. S. Ricardo ${ }^{\mathrm{b}}$ \\ aFaculdade de Educação, Ciências e Letras do Sertão Central, Universidade Estadual do Ceará, 63900-000 Quixadá - CE, Brasil \\ bDepartamento de Química Orgânica e Inorgânica, Centro de Ciências, Universidade Federal do Ceará, 60451-970 Fortaleza - CE, \\ Brasil \\ ${ }^{c}$ Centro de Ciências Exatas e Tecnologia, Universidade Estadual Vale do Acaraú, 62040-730 Sobral - CE, Brasil
}

Recebido em 11/08/2016; aceito em 07/12/2016; publicado na web em 10/02/2017

\begin{abstract}
NEW ALKYL POLYGLYCOSIDES SURFACTANTS BASED ON AMYLOSE EXTRACTED FROM ENGLISH POTATO (SOLANUM TUBEROSUM L.). Alkyl polyglycosides (APGs) are new biodegradable surfactants, non-toxic, synthesized from abundant renewable sources, potentially more appropriate than anionic surfactants. A range of glycosides denoted APG-Cy was synthesized by the Köenig-Knorr reaction using $\mathrm{C}_{10}, \mathrm{C}_{16}$ and $\mathrm{C}_{18}$ alcohols and derived from the degradation of amylose from English potato. The molecular structures of the glycosides were characterized by Nuclear Magnetic Resonance $\left({ }^{1} \mathrm{H}\right.$ and ${ }^{13} \mathrm{C}$ NMR) together with Infrared Spectroscopy (FTIR) and Gel Permeation Chromatography (GPC). The study by NMR allowed the junctions between hydrophilic head groups and hydrophobic tail-groups to be characterized in detail. Conformation of the glycosidic units is ${ }^{4} \mathrm{C}_{1}$ type with a $\beta$ anomeric configuration. The formation of glycosides with five glucose rings linked to the alkyl chain was confirmed by ${ }^{1} \mathrm{H}$ NMR and GPC. Liquid crystals identified by the presence of double melting points were observed by Differential Scanning Calorimetry (DSC) showing thermotropic properties. The surface tension $(\gamma)$ and critical micelle concentration (cmc) were determined by du Noüy method, noting that increasing the length of alkyl chain led to the expected reduction in the cmc. The energies of adsorption and micellization processes calculated from isotherms $\gamma$ versus $\ln \mathrm{c}\left(\mathrm{g} . \mathrm{dm}^{-3}\right)$ indicate cooperativeness of hydrophilic and hydrophobic groups.
\end{abstract}

Keywords: synthesis; characterization; alkyl polyglycosides surfactants; amylose; fatty alcohols.

\section{INTRODUÇÃO}

O consumo de agentes tensoativos não iônicos cresceu no mercado nos últimos 50 anos, devido sua baixa toxicidade e sua vasta utilização em produtos de limpeza, cosméticos, farmacêuticos e alimentícios, atingindo a marca de $30 \%$ de toda a produção de surfactantes em nível mundial, porém, concomitantemente, cresceu a preocupação relacionada com possíveis impactos ambientais. ${ }^{1}$

Surfactantes podem ser derivados de carboidratos e álcoois graxos. ${ }^{2}$ Alquil poliglicosídeos (APGs) são surfactantes não iônicos de interesse especial por serem produzidos a partir de recursos renováveis, ${ }^{3}$ tais como a amilose do amido da batata inglesa, tornando-se substitutos de outros surfactantes que são potencialmente prejudiciais à saúde e ao meio ambiente. ${ }^{4}$

A batata (Solanum tuberosum L.), também conhecida como batatinha ou batata-inglesa, é nativa da América do Sul, da Cordilheira dos Andes. Ocupa o $4^{\circ}$ lugar entre os alimentos mais consumidos do mundo, sendo superada apenas pelo trigo, arroz e milho. No Brasil, é considerada a principal hortaliça, tanto em área cultivada como em preferência alimentar. ${ }^{5-6} \mathrm{O}$ amido é o principal polissacarídeo do milho, arroz, trigo e batata inglesa, que, por sua vez, são as principais fontes de amido. Estes se apresentam na forma de grânulos e diferem significativamente nas propriedades de composição, morfologia, propriedades térmicas, reológicas e retrogradação. ${ }^{7} \mathrm{O}$ amido da batata inglesa exibe maior poder de inchamento, solubilidade, clareza de pasta e viscosidade do que os amidos de trigo, arroz ou milho. As temperaturas de gelatinização

*e-mail: elenirnp@gmail.com dos amidos de trigo e batata inglesa são inferiores as dos amidos de arroz e milho, entretanto, os valores de entalpia de gelatinização são mais elevados, ${ }^{8}$ o que justifica a utilização do amido da batata inglesa para a síntese dos APGs, visto que suas propriedades favorecem as etapas reacionais posteriores da síntese. A amilose foi escolhida como carboidrato para a síntese dos surfactantes por ser um homopolissacarídeo linear do amido, que facilita a sua hidrólise ácida e a torna vantajosa com relação aos heteropolissacarídeos, por apresentar, como único monômero, a glicose. ${ }^{9}$

Além disso, estes surfactantes não iônicos (APGs) apresentam propriedades dermatológicas que favorecem seu uso em cosméticos e higiene pessoal ${ }^{10}$ e são ecologicamente favoráveis (como por exemplo, são biodegradáveis ${ }^{4,11}$ e não bioacumuláveis ${ }^{3}$ ), o que induz sua ampla utilização. Dado que sua propriedade mais favorável é a excelente compatibilidade com a pele em condições típicas de uso, isso leva a uma extensiva utilização como um único surfactante e/ou co-surfactante em formulações para produtos de consumo, ${ }^{3}$ sendo bons substitutos do lauril sulfato de sódio e o dodecil sulfato de sódio, os quais são utilizados em cosméticos mesmo sendo irritantes para a pele. ${ }^{12,13}$ Os grupos cabeças dos APGs consistem geralmente de 1-5 anéis de glicose, enquanto que as caudas são cadeias alquílicas contendo de 6-18 átomos de carbono. APGs com cadeias mais curtas ou mais longas que estas apresentam baixa atividade de superfície, o que inibe sua ampla aplicação. ${ }^{14}$

Detergentes naturais, incluindo APGs como matéria-prima, estão sendo cada vez mais utilizados quando comparados aos seus homólogos derivados sinteticamente, pois são considerados mais seguros para aplicações domésticas. ${ }^{15}$

Alquil poliglicosídeos podem diferenciar-se de acordo com a 
quantidade de anéis de glicose presentes em sua cadeia. A maioria dos APGs apresentados na literatura possuem de um a três anéis de glicose ${ }^{16-19}$ e há ainda trabalhos com os alquil poliglicosídeos superiores sintetizados com cinco ou mais anéis de glicose,${ }^{20}$ no entanto, nenhum com cinco anéis de glicose derivados a partir do amido. Neste trabalho, os APGs sintetizados possuem cinco anéis de glicose por cadeia alquílica, derivados da degradação da amilose como partes hidrofílicas, e utilizam alcoóis com cadeias de 10, 16 e 18 átomos de carbono como partes hidrofóbicas.

\section{PARTE EXPERIMENTAL}

\section{Materiais}

Amido (extraído da batata inglesa); óxido de prata (preparado a partir do nitrato de prata - Carlo Erba); carbonato de prata (Aldrich); piridina (Synth); álcool etílico absoluto (Synth); ácido bromídrico (Merck); anidrido acético (Vetec); ácido acético (Vetec); ácido perclórico (ACS-QM); n-butanol (Synth); bromo líquido (Fluka); fósforo vermelho (Aldrich); sódio metálico (Carlo Erba); decanol (Prosynth); hexadecanol (Vetec); octadecanol (Lancaster); diclorometano (Synth); éter dietílico (Synth); éter de petróleo (Synth); clorofórmio (Synth); gelo seco $\left(\mathrm{CO}_{2}\right)$ (White Martins); gás nitrogênio $\left(\mathrm{N}_{2}\right)$ (White Martins). Todos os reagentes foram utilizados como recebidos, exceto o éter de petróleo e o éter dietílico, que foram previamente secos (destilação na presença de hidreto de cálcio).

A amilose foi isolada a partir do amido extraído da batata inglesa (Solanum tuberosum L.) segundo Whistler, ${ }^{21,22}$ pré-tratada, acetilada e hidrolisada segundo metodologia estabelecida. ${ }^{23-25} \mathrm{E}$ os alquil poliglicosídeos obtidos da amilose ${ }^{26}$ foram sintetizados segundo metodologia estabelecida. ${ }^{27}$ Detalhes da síntese dos APGs da amilose podem ser encontrados no material suplementar. Os APGs sintetizados foram denominados de acordo com sua cadeia alquílica: $\mathrm{APG}-\mathrm{C}_{10}, \mathrm{APG}-\mathrm{C}_{16}$ e $\mathrm{APG}-\mathrm{C}_{18}$.

\section{Determinação do grau de substituição (GS) e percentagem de acetilação $(\mathbf{P A})^{28}$}

A amilose foi acetilada segundo a metodologia de Mark e Mehtretter. ${ }^{29} \mathrm{O}$ percentual de grupos acetila e o grau de substituição dos acetatos de amilose foram determinados por titulomentria conforme Wurzburg (1964). ${ }^{21}$ Amostras pulverizadas dos acetatos (0,5 g) e amilose $(0,5 \mathrm{~g})$ (branco) foram misturadas a $25 \mathrm{~mL}$ de solução aquosa de etanol a $75 \%(\mathrm{v} / \mathrm{v}) \mathrm{cada}$. As amostras foram aquecidas $\left(50^{\circ} \mathrm{C}\right) \mathrm{sob}$ agitação, por $30 \mathrm{~min}$, e em seguida resfriadas. Posteriormente, adicionou-se lentamente $20 \mathrm{~mL}$ de solução padrão de $\mathrm{KOH} 0,5 \mathrm{~mol} \mathrm{~L}^{-1}$ sob agitação em ambas as amostras, deixando-as em repouso por 72 horas. Após este período, titulou-se o excesso de álcali de ambas as amostras (acetatos e branco) com solução padrão de $\mathrm{HCl} 0,5 \mathrm{~mol} \mathrm{~L}^{-1}$, usando fenolftaleína como indicador. Em seguida, ambas as soluções contendo acetatos e branco, respectivamente, permaneceram em repouso por mais duas horas. Na sequência, titulou-se o álcali que estava retido nos novelos dos polímeros, que posteriormente foi liberado das amostras por difusão. O grau de substituição (GS) e a percentagem de acetilação (PA) foram calculados a partir das seguintes expressões: ${ }^{30}$

$\mathrm{PA}=\left[\left\{\mathrm{V}_{\mathrm{mL}}(\right.\right.$ branco $)-\mathrm{V}_{\mathrm{mL}}($ amostra $\left.\left.)\right\} \times \mathrm{M}(\mathrm{HCl}) \times 0,043 \times 100\right] /$ $\mathrm{m}$ amostra $(\mathrm{g})$

Em que: $\mathrm{V}_{\mathrm{mL}}$ branco = soma dos volumes gastos nas duas titulações da amilose após tratamento com $\mathrm{KOH} 0,5 \mathrm{~mol} \mathrm{~L}^{-1}$; $\mathrm{V}_{\mathrm{mL}}$ amostra $=$ soma dos volumes gasto nas duas titulações do triacetato após tratamento com $\mathrm{KOH} \mathrm{0,5} \mathrm{mol} \mathrm{L}^{-1}$; M é a molaridade do $\mathrm{HCl}=0,501 \mathrm{~mol} \mathrm{~L}^{-1}$ e $\mathrm{m}_{\text {amostra }}$ é a massa da amostra.

$$
\mathrm{GS}=(162 \times(\mathrm{PA}) /[4300-42 \times(\mathrm{PA})]
$$

Os valores 162 e 43 são relativos a massa molar da anidro D-glicose e a massa molar do grupo acetil, respectivamente. $\mathrm{O}$ valor 42 refere-se a massa molar do grupo acetil após a saída de um hidrogênio ao se ligar a anidroglicose.

\section{Determinação da massa molar viscosimétrica média $\left(M_{v}\right)$ da amilose $^{31}$}

As medidas de viscosidade foram obtidas em um viscosímetro do tipo Ubbelohde padrão (1-I 71), proveniente da CANNON INSTRUMENTS CO, em um banho termostatizado a $25,0 \pm 0,1{ }^{\circ} \mathrm{C}$, Modelo Q-305.2 da QUIMIS, tendo como base o ASTMD-445. A massa molar da amilose $\left(M_{v}\right)$, foi determinada usando-se a equação de Mark-Houwink, ${ }^{32}$

$$
\begin{aligned}
& {[\eta]=\mathrm{K}\left(\mathrm{M}_{\mathrm{v}}\right)^{\mathrm{a}}} \\
& M_{V}=\sqrt[a]{\frac{[\eta]}{\mathrm{K}}}
\end{aligned}
$$

Em que: $\eta$ é a viscosidade intrínseca, " $K$ " e "a" são constantes que dependem do sistema polímero-solvente. Para o sistema amilose-DMSO ${ }^{33}$ temos $\mathrm{a}=0,70$ e $\mathrm{K}=15,1$ x $10^{-5}$ e $\left(\mathrm{M}_{\mathrm{v}}\right)$ é a massa molar viscosimétrica média. A viscosidade intrínseca foi determinada através das medidas dos tempos de escoamento de solução diluída de amilose em DMSO, em diferentes concentrações. As medidas foram realizadas em triplicatas.

\section{Grau de Polimerização (GP)}

O GP foi obtido através da relação:

$$
\mathrm{GP}=\mathrm{M}_{\mathrm{v}} / \mathrm{M}_{\mathrm{wu}}
$$

Em que: $\mathrm{M}_{\mathrm{v}}$ é a massa molar viscosimétrica média, $\mathrm{M}_{\mathrm{wu}}$ é a massa da unidade monomérica.

Espectroscopia de absorção na região do infravermelho (FTIR)

Os espectros FTIR dos materiais de partida, dos intermediários e dos surfactantes foram obtidos em pastilha de $\mathrm{KBr}$ em um espectrômetro da SHIMADZU FTIR 8300 com varredura de 400 a $4000 \mathrm{~cm}^{-1}$.

\section{Ressonância magnética nuclear de ${ }^{1} \mathrm{H}$ e ${ }^{13} \mathrm{C}\left(\mathrm{RMN}{ }^{1} \mathrm{H}\right.$ e RMN $\left.{ }^{13} \mathbf{C}\right)$}

Os espectros de $\mathrm{RMN}{ }^{13} \mathrm{C}$ no estado sólido (CP-MAS) e RMN ${ }^{1} \mathrm{H}$ e RMN ${ }^{13} \mathrm{C}$ em solução $\left(\mathrm{D}_{2} \mathrm{O}\right.$ como solvente e TMSP como padrão interno) foram obtidos em um equipamento Bruker Avance 300, com transformada de Fourier, funcionando a $300 \mathrm{MHz}$ na frequência do hidrogênio, e a $75 \mathrm{MHz}$ para $\mathrm{RMN}{ }^{13} \mathrm{C}$.

\section{Tensão superficial}

Para as medidas da tensão superficial foi utilizada uma balança de torção da White Elec. Inst. Co. LTD. Todas as medidas foram feitas a $30 \pm 0,1^{\circ} \mathrm{C}$. Parâmetros termodinâmicos foram calculados a partir 
das isotermas de tensão superficial de equilíbrio versus logaritmo natural da concentração. ${ }^{34-38} \mathrm{~A}$ partir das isotermas de $\gamma$ versus $\ln \mathrm{c}$ $\left(\mathrm{g} \cdot \mathrm{dm}^{-3}\right)$ foi determinado o excesso interfacial,

$$
\Gamma=-(1 / \mathrm{RT})(\partial \gamma / \partial \ln \mathrm{c})
$$

Em que: $(\partial \gamma / \partial \ln$ c) é obtido a partir das inclinações das curvas; $R$ é a constante universal dos gases $\left(8,314 \mathrm{~J} \cdot \mathrm{K}^{-1} \mathrm{~mol}^{-1}\right)$; T é a temperatura em Kelvin e a área por molécula (A) é fornecida por:

$$
\mathrm{A}=(1 / \Gamma) \times\left(1 / \mathrm{N}_{\mathrm{A}}\right)
$$

Em que: $\mathrm{N}_{\mathrm{A}}$ é o número de Avogadro e $\pi$ é a pressão interfacial, a qual é encontrada por:

$$
\pi=\left(\gamma-\gamma_{\mathrm{cmc}}\right)
$$

Em que: $\gamma$ é a tensão superficial da água pura e $\gamma_{\mathrm{cmc}}$ é o valor da tensão superficial das soluções dos surfactantes (Figura 12S e Tabela 3).

Os valores de concentração micelar crítica $(\mathrm{cmc})$ podem ser encontrados no ponto em que as duas curvas lineares se cruzam nas isotermas. Os valores do parâmetro crítico de empacotamento (PCE) foi calculado pela equação:

$$
\mathrm{PCE}=v / \mathrm{A} l \mathrm{c}
$$

Na qual: $v$ é o volume da cadeia alquílica; lc é o comprimento para a cadeia alquílica estendida.

As incógnitas v e lc são determinados pelas seguintes expressões:

$$
\begin{gathered}
v=\left(27,4+26,9 \mathrm{nc}^{\prime}\right) \times 10^{-3}\left(\mathrm{~nm}^{3}\right) \\
l \mathrm{c}=0,15+0,1265 \mathrm{nc}(\mathrm{nm})
\end{gathered}
$$

Nas quais: nc' o número de átomos de carbono por cadeia no núcleo micelar.

Parâmetros termodinâmicos tais como, a variação da energia livre de Gibbs $\left(\Delta \mathrm{G}^{\mathrm{o}}\right)$ e a variação da entropia $\left(\Delta \mathrm{S}^{\mathrm{o}}\right)$ para os processos de adsorção e micelização dos surfactantes sintetizados foram determinados empregando as seguintes relações: $:^{38-42}$

$\Delta \mathrm{G}^{\mathrm{o}}$ ads $=\Delta \mathrm{G}^{\mathrm{o}} \mathrm{mic}-\left(\pi_{\mathrm{cmc}} / \Gamma\right)$ e $\Delta \mathrm{G}^{\mathrm{o}}$ mic $=\mathrm{RT}(\ln \mathrm{cmc})$

$\Delta S^{\circ}$ mic $=-\Delta G^{\circ}$ mic $/ T=-R($ ln cmc $)$ e $\Delta S^{\circ}$ ads $=-\Delta G^{\circ}$ ads $/ \mathrm{T}$

Como as medidas de tensão superficial para os surfactantes sintetizados foram realizadas em uma única temperatura (isotermas, $\left.30{ }^{\circ} \mathrm{C}\right), \Delta \mathrm{H}^{\circ}{ }_{\text {mic }}$ foi considerado igual a zero.

$$
\Delta \mathrm{H}_{\text {mic }}^{\mathrm{o}}=-\mathrm{RT} \mathrm{d} \ln (\mathrm{cmc}) / \mathrm{d}(1 / \mathrm{T})
$$

Objetivando estabelecer uma relação entre as estruturas das partes hidrofílica e hidrofóbica dos surfactantes, com suas propriedades físico-químicas em solução aquosa, levou-se em consideração que quantidades termodinâmicas contém informações acerca das contribuições para a transferência da fase aquosa para a micela dos grupos terminal $\left(\mathrm{CH}_{3}\right)$, metilênicos da cadeia alquílica $\left(\mathrm{CH}_{2}\right)$ e grupo cabeça ${ }^{43-46}$ Por isso foram utilizadas as seguintes relações:

$\Delta \mathrm{G}_{\text {ads }}^{\mathrm{o}}=\Delta \mathrm{G}^{\mathrm{o}}$ grupo cabeça $+\Delta \mathrm{G}^{\circ} \mathrm{CH}_{3}+\mathrm{NCH}_{2} \Delta \mathrm{G}^{\circ} \mathrm{CH}_{2}$

$\Delta \mathrm{G}_{\text {mic }}^{\mathrm{o}}=\Delta \mathrm{G}^{\mathrm{o}}$ grupo cabeça $+\Delta \mathrm{G}^{\circ} \mathrm{CH}_{3}+\mathrm{NCH}_{2} \Delta \mathrm{G}^{\circ} \mathrm{CH}_{2}$

$\Delta \mathrm{S}_{\text {mic }}^{\mathrm{o}}=\Delta \mathrm{S}^{\mathrm{o}}$ grupo cabeça $+\Delta \mathrm{S}^{\circ} \mathrm{CH}_{3}+\mathrm{NCH}_{2} \Delta \mathrm{S}^{\circ} \mathrm{CH}_{2}$

$\Delta \mathrm{S}_{\text {ads }}^{\mathrm{o}}=\Delta \mathrm{S}^{\mathrm{o}}$ grupo cabeça $+\Delta \mathrm{S}^{\circ} \mathrm{CH}_{3}+\mathrm{NCH}_{2} \Delta \mathrm{S}^{\circ} \mathrm{CH}_{2}$

\section{Determinação da eficiência e efetividade do surfactante na} redução da tensão superficial

A redução na tensão superficial resulta da adsorção das moléculas surfactantes na interface ar/solução. Estas propriedades de uma molécula surfactante são caracterizadas por sua eficiência e efetividade. A eficiência de um surfactante é definida pelo valor do logarítimo negativo da concentração necessária para reduzir a tensão superficial por $20 \mathrm{dyn} / \mathrm{cm}$, e é designada por $p C 20$.

A efetividade de um surfactante em reduzir a tensão superficial é dada pelo valor de $\pi_{\mathrm{cmc}}$.

\section{Balanço Hidrofílico-Lipofílico (BHL)}

O balanço hidrofílico-lipofílico foi calculado através da equação:

$$
\mathrm{BHL}=20\left(1-\mathrm{M}_{\mathrm{h}} / \mathrm{M}\right)^{47}
$$

Em que: $\mathrm{M}_{\mathrm{h}}$ é a massa molar do grupo hidrofóbico e M é a massa molar do surfactante determinada por GPC.

\section{Cromatografia de permeação em gel (GPC)}

A massa molar média de todos os surfactantes foi estimada através de cromatografia de permeação em gel (GPC). As soluções preparadas $(0,4 \%)$ foram injetadas $(0,5 \mu \mathrm{L})$, com fluxo de $1,0 \mathrm{~mL}$ por minuto em sistema de duas colunas em série da Phenomenex, do tipo Phenogel LINEAR/MIXED 5U com 7,80 x $300 \mathrm{~mm}$, sendo utilizado tolueno como fase móvel à temperatura ambiente. A curva de calibração foi construída utilizando-se padrões de poliisopreno com massas molares em intervalo de grandeza de $1.130 \mathrm{~g} \mathrm{~mol}^{-1}$ a $2.160 .000 \mathrm{~g} \mathrm{~mol}^{-1}$. Foi utilizado cromatógrafo da Shimadzu LC-10AD, com detectores de índice de refração (RID-6A) e ultravioleta (UV-VIS SPD-10AV).

\section{Análise térmica (TGA e DSC)}

Medidas de DSC e TGA foram realizadas em equipamentos da SHIMADZU (DSC-50 e TGA-50) em atmosfera de hélio a uma taxa de aquecimento de $10{ }^{\circ} \mathrm{C} \mathrm{min}^{-1}$ na faixa de temperatura de $25-450{ }^{\circ} \mathrm{C}$ para o DSC e $25-700^{\circ} \mathrm{C}$ para o TGA.

\section{RESULTADOS E DISCUSSÃO}

\section{FT-IR para a amilose e seus derivados ${ }^{48}$}

Os modos vibracionais da amilose são conhecidos por serem muitos semelhantes àqueles do amido e glicose e ocorrem aproximadamente nas mesmas frequências (Figura 1S). A acetilação da amilose foi confirmada pelo aparecimento de quatro bandas características dos grupos acetilas $\left(1751 \mathrm{~cm}^{-1}, \mathrm{vC}=\mathrm{O} ; 1365 \mathrm{~cm}^{-1}, \mathrm{vCH}\right.$ do grupo $\mathrm{CH}_{3} ; 1234 \mathrm{~cm}^{-1}, \mathrm{vC}-\mathrm{O}$ e $\left.1036 \mathrm{~cm}^{-1}, \mathrm{vC}-\mathrm{O}\right)$. A banda fraca em torno de $3500 \mathrm{~cm}^{-1}$ ocorreu, provavelmente, devido a grupos $\mathrm{OH}$ remanescentes na molécula, considerando-se que o grau de substituição (GS) do polímero foi de $\sim 2,8$ (Figura 1).

A entrada da cadeia alquílica foi confirmada observando-se o alargamento das bandas, na faixa de $1100-1031 \mathrm{~cm}^{-1}$, decorrente provavelmente da sobreposição, devido ao desdobramento das bandas C-O-C do éter glicosídico formado (Figura 1(d)). O deslocamento observado na frequência em $911 \mathrm{~cm}^{-1}$ do carbono anomérico também justifica a presença da cadeia alquílica. A desacetilação foi confirmada pela comparação dos espectros da Figura 1(b-c) com o espectro da Figura 1(d), sendo constatado que houve o desaparecimento das bandas características dos grupos acetilas, $v(C=O)$ e $v(C-O)$ e o 


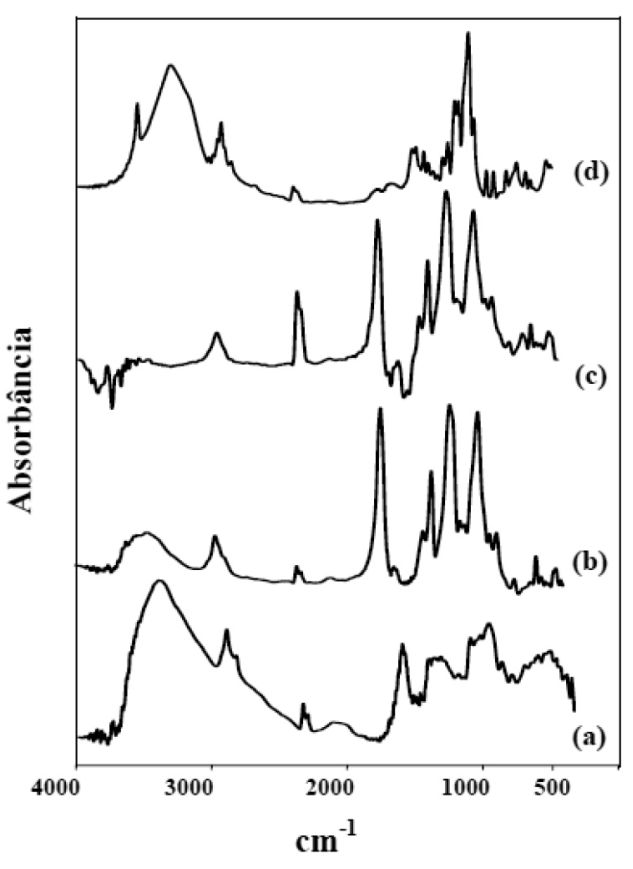

Figura 1. FTIR: (a) Amilose, (b) amilose acetilada, (c) amilose acetilada e hidrolisada e (d) decil poliglicosídeo $\left(A P G-C_{10}\right)$

aparecimento da banda na região de $3400 \mathrm{~cm}^{-1}$, atribuída ao $v(\mathrm{OH})$ da hidroxila de resíduos de glicose. Em $2900 \mathrm{~cm}^{-1}$, observou-se o $v\left(\mathrm{CH}_{2}\right)$ da cadeia alquílica.

\section{Grau de substituição (GS) e percentagem de acetilação (PA $)^{49}$}

A percentagem de acetilação foi calculada obtendo-se um valor de $\sim 43 \%$. Por outro lado, o grau de substituição (GS) foi calculado a partir da percentagem de acetilação. Portanto, o GS foi de $\sim 2,8$.

\section{Determinação da massa molar viscosimétrica média $\left(M_{v}\right)^{50}$}

O gráfico de determinação da viscosidade intrínseca da amilose a $25^{\circ} \mathrm{C}$ é mostrado na Figura $2 \mathrm{~S}$. A massa molar viscosimétrica foi determinada pela equação de Mark-Houwink (Equação (3)). A viscosidade intrínseca determinada foi $1,61 \mathrm{dL} / \mathrm{g}$. Para amilose, $\mathrm{M}_{\mathrm{v}}=$ $5,67 \times 10^{5} \mathrm{~g} \mathrm{~mol}^{-1}$, para a amilose acetilada $\mathrm{M}_{\mathrm{v}}=9,76 \times 10^{5} \mathrm{~g} \mathrm{~mol}^{-1}$.

\section{Grau de Polimerização (GP)}

O GP foi obtido através da equação (5). Os valores encontrados foram 3.389 e 3.500 para amilose acetilada e amilose, respectivamente.

\section{Determinação da massa molar dos surfactantes por GPC (11-52 $^{5}$}

Observando-se os cromatogramas de GPC para os surfactantes sintetizados (Figuras 3S-5S) nota-se uma distribuição de massa bimodal para os três APGs, sendo o ombro a 10,40 mL atribuídos aos respectivos alquil glicosídeos acetilados e o valor em aproximadamente 10,60 $\mathrm{mL}$ referente aos alquil glicosídeos. Os valores de $\mathrm{M}_{\mathrm{pk}}$ e GP que correspondem ao número de unidades de glicose por molécula surfactante são mostrados na Tabela 1. Observou-se, também, no cromatograma referente ao APG- $\mathrm{C}_{10}$, a presença de outro ombro a 11,10 mL atribuídos à maltose (açúcar comum em hidrolisados da amilose) que pode ter sido formada durante a hidrólise da amilose e que não foi removida completamente durante as etapas de purificação (Figura 3S).
Tabela 1. Valores das massas molares para os alquil glicosídeos (APGs) derivados da amilose (APG-C 10 , APG- $\mathrm{C}_{16}$ e APG-C $\mathrm{C}_{18}$ )

\begin{tabular}{ccc}
\hline Amostras & $\mathrm{M}_{\mathrm{pk}}\left(\mathrm{g} \mathrm{mol}^{-1}\right)$ & $\mathrm{DP}_{\mathrm{méd}}=\left(\mathrm{M}_{\mathrm{pk}}-\mathrm{M}_{\mathrm{h}}\right) / \mathrm{M}_{\mathrm{wu}}$ \\
\hline APG-C & 955 & 5,0 \\
APG-C $_{16}$ & 1039 & 5,0 \\
APG-C $_{18}$ & 1067 & 5,0 \\
\hline
\end{tabular}

Obs: $\mathrm{M}_{\mathrm{pk}}=$ massa molar de pico, $\mathrm{M}_{\mathrm{h}}=$ massa molar do grupo hidrofóbico, $\mathrm{M}_{\mathrm{wu}}=$ massa molar da unidade monomérica $=162$.

\section{Análise Térmica (TGA e DSC) $)^{53}$}

TGA para amido, amilose e seus derivados ${ }^{54-56}$

As curvas TGA e DTG da amilose e surfactantes derivados da amilose são mostrados nas Figuras 6S-11S. A amilose analisada apresentou duas principais etapas de perda de massa. Observou-se na DTG da amilose da batata um evento em torno de $58,5^{\circ} \mathrm{C}$, um segundo evento em torno de $322^{\circ} \mathrm{C}$ e um terceiro evento em 375,5 ${ }^{\circ} \mathrm{C}$ (Figura 6S). A etapa de decomposição em torno de $40^{\circ} \mathrm{C}$ a $66^{\circ} \mathrm{C}$ para todos os materiais analisados foi atribuída à perda de água por volatilização. A amilose triacetilada, bem como o seu hidrolisado, apresentaram praticamente apenas um evento principal de decomposição em torno de $370^{\circ} \mathrm{C}$ (Figuras $7 \mathrm{~S}$ e $8 \mathrm{~S}$ ). Observou-se também que estes derivados da amilose perderam pouca água na faixa de temperatura acima considerada $\left(40{ }^{\circ} \mathrm{C}\right.$ a $\left.66^{\circ} \mathrm{C}\right)$, o que pode ser justificado pelo aumento da lipofilicidade da amilose pela entrada dos grupos decorrente da acetilação, devido provavelmente à ruptura da estrutura de dupla hélice da amilose para formar o material acetilado de hélice simples que cristaliza retendo menores quantidades de água. Tal como observado na literatura, ${ }^{57-59}$ a temperatura inicial de decomposição da amilose foi deslocada para um maior valor $\left(\mathrm{de} 322^{\circ} \mathrm{C}\right.$ para $\left.370{ }^{\circ} \mathrm{C}\right)$ no material acetilado. Os surfactantes APG- $\mathrm{C}_{16}$ e APG- $\mathrm{C}_{18}$ apresentaram decomposição em um único estágio (Figuras $10 \mathrm{~S}$ e $11 \mathrm{~S}$ ). O APG- $\mathrm{C}_{10}$, entretanto, mostrou dois estágios de decomposição (Figura 9S). A Tabela 2 apresenta o comportamento térmico para as principais etapas de decomposição para a amilose e para os derivados da amilose observados nas curvas DTG.

Tabela 2. Temperatura de pico $\left(\mathrm{T}_{\mathrm{p}}\right)$ para a amilose, amilose acetilada, amilose acetilada e hidrolisada e alquil poliglicosídeos derivados da amilose indicadas nas curvas DTG, relacionados aos principais eventos

\begin{tabular}{ccccc}
\hline \multirow{2}{*}{ Amostras } & \multicolumn{3}{c}{ Principais eventos da decomposição } & \\
\cline { 2 - 4 } & $1^{\circ}$ & $2^{\circ}$ & $3^{\circ}$ & Resíduo \\
\cline { 2 - 3 } & $\mathrm{T}_{\mathrm{p}}\left({ }^{\circ} \mathrm{C}\right)$ & $\mathrm{T}_{\mathrm{p}}\left({ }^{\circ} \mathrm{C}\right)$ & $\mathrm{T}_{\mathrm{p}}\left({ }^{\circ} \mathrm{C}\right)$ & \\
\hline AmB $^{*}$ & 58,5 & 322,0 & 375,1 & 15,06 \\
AmAc* $^{*}$ & 41,6 & 369,5 & - & 15,77 \\
AmAcH $^{*}$ & - & 370,4 & - & 15,78 \\
APG- $\mathrm{C}_{10}$ & 65,8 & 190,1 & 297,6 & 9,98 \\
APG- $\mathrm{C}_{16}$ & - & 296,4 & - & 9,75 \\
APG- $\mathrm{C}_{18}$ & - & 303,9 & - & 7,23 \\
\hline
\end{tabular}

${ }^{*} \mathrm{AmB}=$ amilose isolada da batata inglesa, $\mathrm{AmAc}=$ amilose acetilada, $\mathrm{AmAcH}$ $=$ amilose acetilada e hidrolisada.

DSC para a amilose e seus derivados ${ }^{60}$

As curvas de DSC para a amilose, amilose acetilada, oligo acetatos e alquil poliglicosídeos são mostradas nas Figuras 12S-17S. A curva para a amilose da batata, em geral, exibe três transições endotérmicas e uma exotérmica. A primeira transição endotérmica $\left(81^{\circ} \mathrm{C}\right)$ é atribuída ao fluxo de água no material, intumescimento, 
Tabela 3. Comportamento Calorimétrico para o amido, amilose e para os derivados da amilose (amilose acetilada, oligo acetatos e alquil glicosídeos)

\begin{tabular}{|c|c|c|c|c|c|c|c|c|c|c|}
\hline \multirow{3}{*}{ Amostras } & \multicolumn{10}{|c|}{ Transições físicas } \\
\hline & \multicolumn{2}{|c|}{$1^{\mathrm{a}}$} & \multicolumn{2}{|c|}{$2^{\mathrm{a}}$} & \multicolumn{2}{|c|}{$3^{\mathrm{a}}$} & \multicolumn{2}{|c|}{$4^{a}$} & \multicolumn{2}{|c|}{$5^{\mathrm{a}}$} \\
\hline & $\mathrm{T}_{\mathrm{p}}\left({ }^{\circ} \mathrm{C}\right)$ & $\mathrm{E}(\mathrm{J} / \mathrm{g})$ & $\mathrm{T}_{\mathrm{p}}\left({ }^{\circ} \mathrm{C}\right)$ & $\mathrm{E}(\mathrm{J} / \mathrm{g})$ & $\mathrm{T}_{\mathrm{p}}\left({ }^{\circ} \mathrm{C}\right)$ & $\mathrm{E}(\mathrm{J} / \mathrm{g})$ & $\mathrm{T}_{\mathrm{p}}\left({ }^{\circ} \mathrm{C}\right)$ & $\mathrm{E}(\mathrm{J} / \mathrm{g})$ & $\mathrm{T}_{\mathrm{p}}\left({ }^{\circ} \mathrm{C}\right)$ & $\mathrm{E}(\mathrm{J} / \mathrm{g})$ \\
\hline $\mathrm{AmB}$ & 81 & $-582,8$ & 288,0 & $-60,1$ & 297,0 & $-121,0$ & 350,5 & 85,6 & - & - \\
\hline AmAc & 51,9 & $-76,4$ & 173,2 & $-15,0$ & 185,7 & 28,8 & 297,7 & $-16,9$ & 373,6 & 30,0 \\
\hline $\mathrm{AmAcH}$ & 66,9 & $-170,9$ & 163,4 & $-60,8$ & 183,5 & 83,0 & 298,4 & $-43,0$ & 324,2 & 9,2 \\
\hline APG- $C_{10}$ & 33 & $-8,7$ & 108 & $-102,5$ & 267,0 & $-744,0$ & - & - & - & - \\
\hline APG- $C_{16}$ & 55,8 & $-9,7$ & 161 & $-105,8$ & 273,0 & $-422,0$ & 306,0 & $-37,6$ & - & - \\
\hline APG- $\mathrm{C}_{18}$ & 38,9 & $-7,7$ & 165 & $-105,6$ & 215,9 & 12,2 & 307,9 & -880 & - & - \\
\hline
\end{tabular}

quebra de ligações de hidrogênio e/ou desorganização da estrutura semicristalina dos materiais (amilose). (Figura 12S). A segunda transição endotérmica $\left(288^{\circ} \mathrm{C}\right)$ corresponde à fusão do material enquanto as outras transições são referentes às etapas de decomposição do material (Figura 12S). As curvas de DSC para o triacetato de amilose (Figura 13S) e seus oligo acetatos derivados da hidrólise (Figura 14S) apresentaram de quatro a cinco eventos endotérmicos. A primeira transição endotérmica para ambos os materiais, é atribuída ao fluxo de água e, principalmente, à desorganização da estrutura dos cristais. Os dois derivados (triacetato de amilose e oligo acetatos) apresentaram uma transição endotérmica em torno de $300^{\circ} \mathrm{C}$, relativa à temperatura de fusão, o que representa uma característica de material acetilado. ${ }^{61}$

Para os surfactantes derivados da amilose, verificaram-se as transições com temperaturas relativamente baixas, que são características de surfactantes $\left(33^{\circ} \mathrm{C}-55,8^{\circ} \mathrm{C}\right.$ perca de água por volatilização e $108^{\circ} \mathrm{C}-165^{\circ} \mathrm{C}$ - ponto de fusão). Também foram observadas transições em torno de $267^{\circ} \mathrm{C}$ a $307,9^{\circ} \mathrm{C}$, características da decomposição dos materiais (Figuras 15S-17S). A Tabela 3 apresenta o comportamento térmico das principais transições bem como os valores das energias envolvidas para o amido, amilose e para os derivados da amilose (amilose acetilada, oligo acetatos e alquil poliglicosídeos).

\section{Ressonância Magnética Nuclear (RMN)}

RMN para amilose e para os alquil poliglicosídeos derivados da amilose

A Figura 2 mostra uma estrutura genérica dos surfactantes $(\mathrm{n}=$ 1-5 e $\mathrm{x}=10-18)$. As letras $(\mathrm{a}-\mathrm{d})$ e os números $(1-6)$ identificam os diferentes prótons e carbonos dos espectros RMN ${ }^{1} \mathrm{H}$ e $\mathrm{RMN}{ }^{13} \mathrm{C}$ para os surfactantes sintetizados.

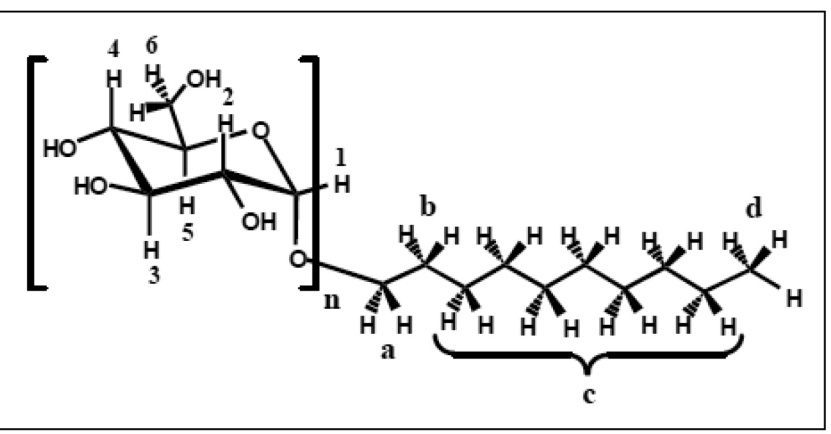

Figura 2. Estrutura genérica de um surfactante alquil poliglicosídico

$R M N{ }^{13} \mathrm{C}$ para a amilose e seus derivados

Nas Figuras 3 e 4 são mostrados os espectros RMN ${ }^{13} \mathrm{C}(\mathrm{CP} /$ MAS) da amilose e amilose acetilada.

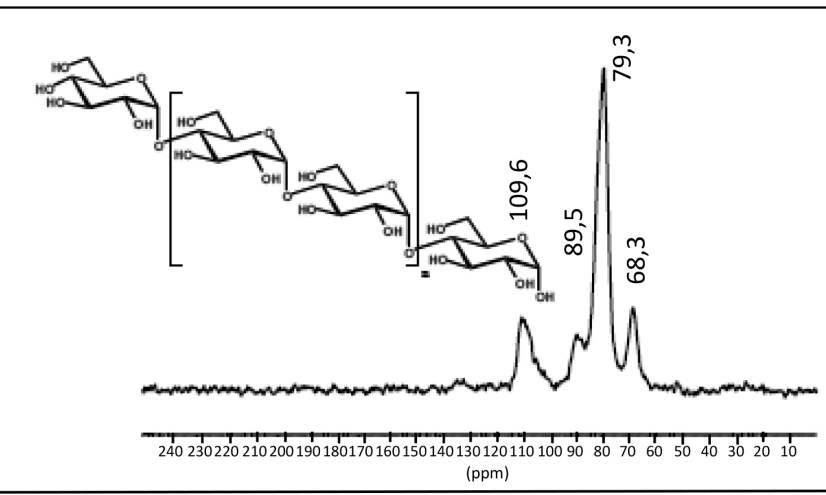

Figura 3. $R M N{ }^{13} C(C P / M A S)$ da amilose da batata inglesa

Na Figura 3 os sinais em 109,6 ppm e em 89,5 ppm foram atribuídos ao C-1 e C-4 da amilose respectivamente. O sinal em 68,3 ppm foi atribuído ao C-6 hidroximetilênico. Associou-se o sinal largo em torno de 79,3 ppm aos C-2, C-3 e C-5.

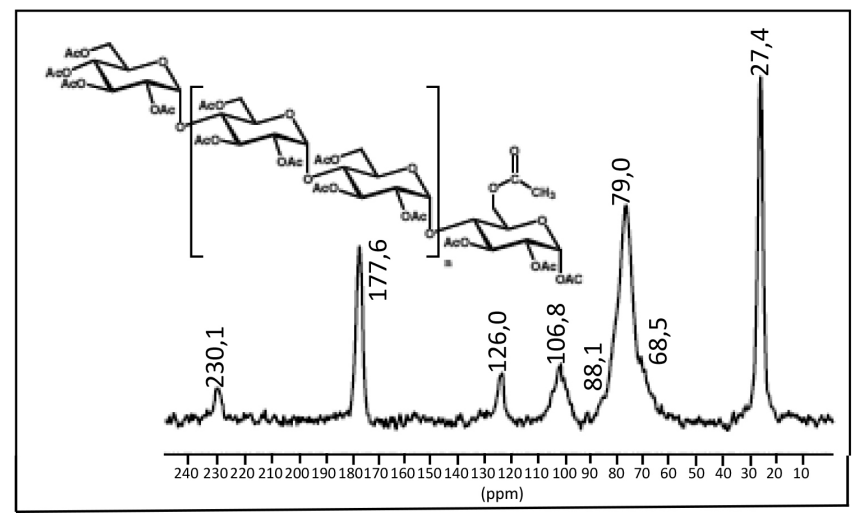

Figura 4. $R M N^{13} \mathrm{C}(\mathrm{CP} / \mathrm{MAS})$ da amilose acetilada

Os sinais em 177,6 ppm e 27,4 ppm foram atribuídos, respectivamente, aos grupos $\mathrm{C}=\mathrm{O}$ e $\mathrm{CH}_{3}$ dos grupos acetilas (Figura 4), confirmando a acetilação da amilose e reforçando os resultados obtidos por FTIR. Os sinais em 230,1 ppm e 126,0 ppm nos espectros acima mencionados foram atribuídos às bandas laterais rotacionais da carbonila. O sinal em $88,1 \mathrm{ppm}$ foi atribuído ao C-4, enquanto que o sinal em 68,5 ppm foi atribuído ao C-6 e o sinal largo em 79,0 ppm foi atribuído aos C-2, C-3 e C-5. A Tabela 4 mostra os deslocamentos químicos $\left(\mathrm{RMN}{ }^{13} \mathrm{C}\right)$ característicos da amilose, amilose triacetato e dos malto oligossacarídeos peracetilados obtidos após hidrólise do triacetato. 
Tabela 4. Deslocamento químico de $\mathrm{RMN}{ }^{13} \mathrm{C}$ em DMSO para amilose e em $\mathrm{CDCl}_{3}$ para seus derivados

\begin{tabular}{cccccccccc}
\hline \multirow{2}{*}{ Amostras } & \multicolumn{1}{c}{ Deslocamento químico (ppm) } \\
\cline { 2 - 9 } & C-1 & C-2 & C-3 & C-4 & C-5 & C-6 & $\mathrm{CH}_{3}$ & C=O \\
\hline AmB & 105,5 & 77,4 & 78,4 & 84,2 & 77,0 & 65,8 & - & - \\
AmAc & 96,2 & 70,8 & 72,0 & 73,8 & 69,5 & 62,9 & 20,9 \\
AmAcH & 96,0 & 70,8 & 72,0 & 73,7 & 69,5 & 62,9 & 20,9 & 170,5 \\
\hline
\end{tabular}

Tabela 5. Deslocamentos químicos de RMN ${ }^{1} \mathrm{H}$ para os surfactantes da amilose (APG-Cy $)$

\begin{tabular}{ccccccc}
\hline \multirow{2}{*}{ Composto } & \multicolumn{7}{c}{ Deslocamento químico (ppm) } \\
\cline { 2 - 7 } & $\mathrm{C}_{\mathbf{1}} \mathbf{H}_{\mathbf{1}}$ & $\mathrm{C}-\mathbf{H}$ & $\mathrm{C}_{6} \mathbf{H}_{\mathbf{6}}$ & $\mathrm{C}_{\mathrm{c}} \mathbf{H}_{\mathbf{c}}$ & $\mathrm{C}_{\mathrm{b}} \mathbf{H}_{\mathbf{b}}$ & $\mathrm{C}_{\mathrm{a}} \mathbf{H}_{\mathbf{a}}$ \\
\hline APG-C & 4,12 & 3,65 & 3,20 & 0,77 & 1,67 & 1,50 \\
APG-C $_{16}$ & 4,14 & 3,50 & 3,31 & 0,82 & 1,66 & 1,67 \\
APG-C $_{18}$ & 4,19 & 3,42 & 3,32 & 0,83 & 1,77 & 1,64 \\
\hline
\end{tabular}

$R M N{ }^{1} \mathrm{H}$ e ${ }^{13} \mathrm{C}$ para os surfactantes derivados da amilose.

A Figura 5 representa o espectro de $\mathrm{RMN}{ }^{1} \mathrm{H}$ do decil oligoglicosídeo derivado da amilose, onde podemos observar detalhes das regiões referentes ao carbono anomérico (4,31 ppm), anel glicosídico $(3,28-3,80 \mathrm{ppm})$ e cadeia alquílica lateral $(0,82-1,61 \mathrm{ppm})$. As Figuras 6 e 7 apresentam os espectros de $\mathrm{RMN}{ }^{13} \mathrm{C}$ e $\mathrm{RMN}{ }^{13} \mathrm{C}$ (DEPT)

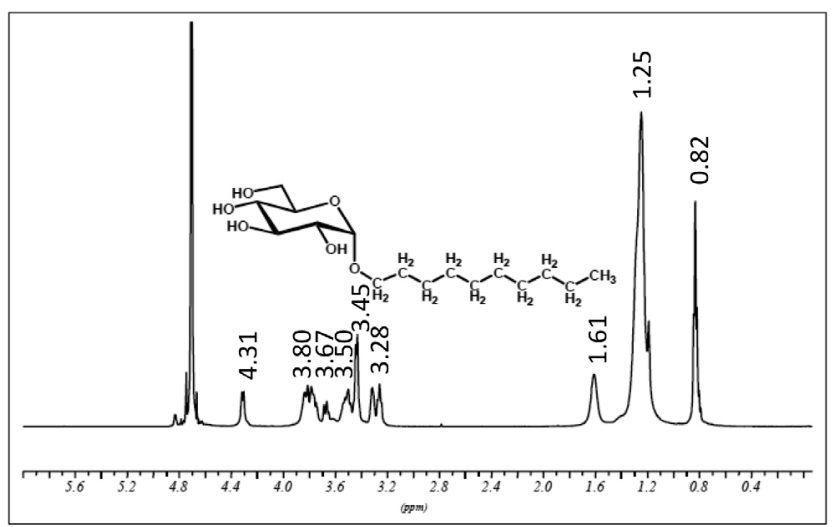

Figura 5. RMN ${ }^{l}$ H para o decil oligoglicosídeo derivado da amilose

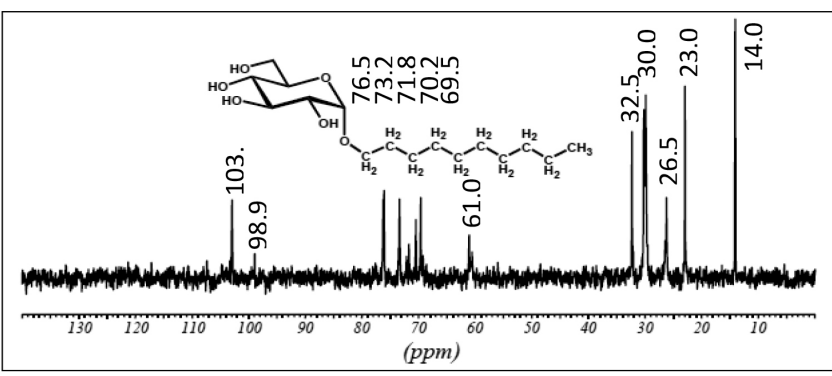

Figura 6. $R M N{ }^{13} \mathrm{C}$ para o decil oligoglicosídeo derivado da amilose

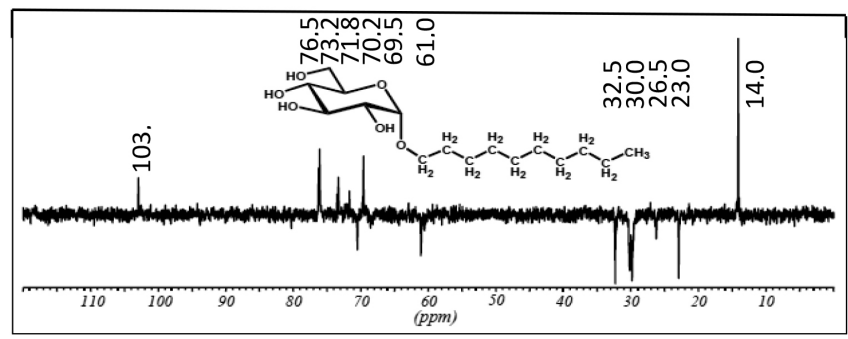

Figura 7. $R M N^{13} C(D E P T)$ para o decil oligoglicosídeo derivado da amilose do decil oligoglicosídeo derivado da amilose. Os deslocamentos químicos nestes espectros foram: $(14,0-32,5 \mathrm{ppm})$ carbonos da cadeia alquílica, $(61,0$ - 76,5 ppm) carbonos dos anéis de glicose e (98,9 - 103,0 ppm) referente ao carbono anomérico. Os deslocamentos químicos correspondentes ao espectro $\mathrm{RMN}{ }^{1} \mathrm{H}$ dos três surfactantes são mostrados na Tabela 5 e podem ser comparados aos deslocamentos químicos observados nos espectros dos surfactantes preparados a partir da glicose.

\section{Comportamento de agregação em solução}

Tensão superficial ${ }^{62}$

As isotermas de tensão superficial de equilíbrio $(\gamma)$ versus ln c (cé aconcentração em $\mathrm{g} \mathrm{dm}^{-3}$ ) exibiram o mesmo perfil de comportamento. Para ilustrar, na Figura 18S é mostrado o gráfico para os surfactantes preparados a partir da amilose. A Tabela 6 apresenta o comportamento em solução aquosa dos surfactantes sintetizados. Os valores de tensão superficial apresentados na tabela podem ser comparados com o valor da água pura $71,5 \mathrm{mN} \mathrm{m}^{-1}$. Os valores da $\mathrm{cmc}\left(\mathrm{g} \mathrm{dm}^{-3}\right)$ e tensão superficial $\gamma\left(\mathrm{mN} \mathrm{m}^{-1}\right)$ para os surfactantes foram: APG-C $\mathrm{C}_{10}(0,31$; $40,54)$, APG- $\mathrm{C}_{16}(0,24 ; 38,20)$, APG- $\mathrm{C}_{18}(0,20 ; 35,60)$. Para os surfactantes sintetizados foram calculados, a partir das isotermas de tensão superficial de equilíbrio versus logaritmo natural da concentração, o excesso da concentração na superfície $(\Gamma)$, a pressão interfacial $(\pi)$ e a área por molécula $(\mathrm{A})^{63}$ através das equações (6) - (8). Os valores de $\Gamma, \mathrm{A}, \pi$ e cmc são mostrados na Tabela 6 .

$\mathrm{O}$ grupo cabeça na série APG- $\mathrm{C}_{\mathrm{y}}$ (em que y pode ser 10,16 ou 18) é volumoso (cinco unidades de glicose) e era de se esperar que A aumentasse. Entretanto, o contrário foi observado. Isto pode ser justificado devido a um empacotamento mais efetivo das moléculas dos malto oligossacarídeos nos surfactantes sintetizados, devido às interações maiores entre estes grupos do que aquelas nos grupos hidrofílicos menores e, por conseguinte, menor hidratação relativa dos mesmos. Resultados semelhantes foram obtidos por Plou e col. ${ }^{64}$

\section{Eficiência e efetividade do surfactante na redução da tensão superficial}

Os dados de $p C 20$ e $\pi_{\text {cmc }}$ estão registrados na Tabela 7 .

Como esperado, a eficiência na redução da tensão superficial na interface ar/solução, devido à agregação das espécies anfifílicas, mostra um aumento linear com o aumento da cadeia hidrocarbônica lateral, enquanto que a efetividade aumenta com o aumento da quantidade das espécies adsorvidas, $\Gamma$ (Figura 19S). Os valores de $p C 20$ e $\pi_{\text {cmc }}$ dos surfactantes sintetizados foram maiores com o 
Tabela 6. Valores de tensão superficial $\left(\gamma_{\mathrm{cmc}}\right)$, $\mathrm{cmc}$ (expresso em $\mathrm{g} \mathrm{dm}^{-3}$, mol L $\mathrm{L}^{-1}$ e fração molar), concentração superficial das moléculas de surfactante adsorvidas na superfície $(\Gamma)$, área da superfície por molécula $(\mathrm{A})$ e pressão superficial $(\pi)$ para os alquil glicosídeos sintetizados

\begin{tabular}{cccccccc}
\hline Amostras & $\begin{array}{c}\gamma \\
\left(\mathrm{mN} \mathrm{m}^{-1}\right)\end{array}$ & $\begin{array}{c}\mathrm{cmc} \\
\left(\mathrm{g} \mathrm{dm}^{-3}\right)\end{array}$ & $\begin{array}{c}\mathrm{cmc} \times 10^{4} \\
\left(\mathrm{~mol} \mathrm{~L}^{-1}\right)\end{array}$ & $\chi_{\mathrm{cmc}} \times 10^{6}$ & $\begin{array}{c}\Gamma \times 10^{6} \\
\left(\mathrm{~mol} \mathrm{~m}^{-2}\right)\end{array}$ & $\begin{array}{c}\pi \\
\left(\mathrm{mN} \mathrm{m}^{-1}\right)\end{array}$ & $\begin{array}{c}\mathrm{A} \\
\left(\mathrm{nm}^{2}\right)\end{array}$ \\
\hline APG- $\mathrm{C}_{10}$ & 40,54 & 0,31 & 3,2 & 5,77 & 2,87 & 30,96 & 0,58 \\
APG- $C_{16}$ & 38,20 & 0,24 & 2,3 & 4,14 & 3,32 & 33,30 & 0,50 \\
APG- $_{18}$ & 35,60 & 0,20 & 1,9 & 3,42 & 3,69 & 35,90 & 0,45 \\
\hline
\end{tabular}

Tabela 7. Valores encontrados para a eficiência $\left(p C_{20}\right)$ e efetividade $\left(\pi_{\mathrm{cmc}}\right)$ para os surfactantes sintetizados

\begin{tabular}{cccc}
\hline Surfactantes & Eficiência $\left(p \mathrm{C}_{20}\right)$ & Efetividade $\left(\pi_{\mathrm{cmc}}\right)$ & $\Gamma\left(\mathrm{mol} \mathrm{m}^{-2}\right) \times 10^{6}$ \\
\hline APG- $\mathrm{C}_{10}$ & 3,5 & 30,96 & 2,87 \\
APG- $\mathrm{C}_{16}$ & 3,7 & 33,30 & 3,32 \\
APG- $\mathrm{C}_{18}$ & 3,8 & 35,90 & 3,69 \\
\hline
\end{tabular}

aumento do tamanho da cadeia alquílica. Isso indica que tanto a eficiência quanto a efetividade são influenciadas pelo tamanho da parte hidrofóbica.

\section{Determinação do Parâmetro Crítico de Empacotamento (PCE)}

A curvatura preferida da interface é governada pelos valores relativos da área $\mathrm{A}$ do grupo cabeça e área efetiva $\mathrm{A}^{\prime}$ da cauda $(v / l \mathrm{c}){ }^{65}$ Os parâmetros críticos de empacotamento para as séries de surfactantes sintetizados foram calculados de acordo com Söderberg e col. (equações (9) - (11) $)^{66}$ com o objetivo da predição da geometria dos agregados e tipos de emulsões formadas. De acordo com os valores de PCE, a estrutura esperada para os agregrados é de micelas cilíndricas relativamente grandes ou em formato de bastão e o tipo de emulsão esperada para os mesmos é do tipo óleo em água (O/A). Os valores de PCE e os valores da relação entre A e A' estão apresentados na Tabela 8.

\section{Determinação dos parâmetros termodinâmicos para os processos} de adsorção e micelização

Parâmetros termodinâmicos, tais como a variação da energia livre de Gibbs $\left(\Delta \mathrm{G}^{\circ}\right)$ e a variação da entropia $\left(\Delta \mathrm{S}^{\circ}\right)$ para os processos de adsorção e micelização dos surfactantes sintetizados, foram determinados empregando as equações (12) - (14). Os dados estão sumarizados na Tabela 9 .

Gráficos dos dados termodinâmicos $\left(\Delta \mathrm{G}^{\mathrm{o}} \mathrm{e} \Delta \mathrm{S}^{\mathrm{o}}\right)$ vs $\mathrm{NCH}_{2}$ e log cmc vs Nc foram plotados observando-se uma relação linear para os surfactantes sintetizados (Figuras 20S(a,b); 21S(a,b) e 22S). Pode ser observado das expressões anteriores que informações acerca da contribuição dos grupos cabeças podem ser obtidas a partir da intersecção (coeficiente linear) das curvas com o eixo $\mathrm{Y}$, a qual contém ambas as contribuições dos grupos cabeça e $\mathrm{CH}_{3}$. Uma vez que as quantidades termodinâmicas para o grupo $\mathrm{CH}_{3}$ são independentes do comprimento da cadeia em uma série homóloga de surfactantes, a intersecção reflete essencialmente o efeito da transferência do grupo cabeça da solução à interface ar/solução, enquanto que informações acerca das contribuições dos grupos $\mathrm{CH}_{2}$ podem ser obtidas a partir dos coeficientes angulares das referidas curvas. As relações obtidas a partir de regressão linear para os surfactantes sintetizados estão sumarizadas na Tabela 10.

Os dados claramente indicam que a transferência dos grupos cabeça é favorável para os surfactantes sintetizados. $\Delta \mathrm{G}^{\mathrm{o}}$ grupo cabeça $+\Delta \mathrm{G}^{\mathrm{o}} \mathrm{CH}_{3}$ para a adsorção $\left(-41,0 \mathrm{~kJ} \mathrm{~mol}^{-1}\right)$ é mais favorável do que

Tabela 8. Relação entre A e A' para surfactantes sintetizados e sua correlação com o tipo de emulsão esperada

\begin{tabular}{cccccc}
\hline Surfactantes & PCE & $\mathrm{A}\left(\mathrm{nm}^{2}\right)$ & $\mathrm{A}^{\prime}\left(\mathrm{nm}^{2}\right)$ & A/A' & Tipo de emulsão esperada \\
\hline APG- $\mathrm{C}_{10}$ & 0,36 & 0,58 & 0,21 & 2,8 & Emulsões de óleo em água \\
APG- $\mathrm{C}_{16}$ & 0,42 & 0,50 & 0,21 & 2,4 & \\
APG- $\mathrm{C}_{18}$ & 0,46 & 0,45 & 0,21 & 2,1 & \\
\hline
\end{tabular}

Tabela 9. Parâmetros termodinâmicos para as etapas de adsorção e micelização envolvidas no processo de auto associação para as espécies anfifílicas sintetizadas

\begin{tabular}{|c|c|c|c|c|c|}
\hline Surfactantes & $\mathrm{cmc} \times 10^{4}\left(\mathrm{~mol} \mathrm{~L}^{-1}\right)$ & $\Delta \mathrm{G}_{\mathrm{ads}}^{\mathrm{o}}\left(\mathrm{kJ} \mathrm{mol}^{-1}\right)$ & $\Delta \mathrm{G}^{\mathrm{o}}{ }_{\text {mic }}\left(\mathrm{kJ} \mathrm{mol}^{-1}\right)$ & $\Delta \mathrm{S}_{\text {ads }}^{\mathrm{o}}\left(\mathrm{kJ} \mathrm{mol}^{-1} \mathrm{~K}^{-1}\right)$ & $\Delta \mathrm{S}_{\text {mic }}^{\mathrm{o}}\left(\mathrm{kJ} \mathrm{mol}^{-1} \mathrm{~K}^{-1}\right)$ \\
\hline APG- $C_{10}$ & 3,2 & $-41,20$ & $-30,40$ & 0,1360 & 0,1000 \\
\hline APG- $\mathrm{C}_{16}$ & 2,3 & $-41,35$ & $-31,34$ & 0,1367 & 0,1035 \\
\hline APG- $\mathrm{C}_{18}$ & 1,9 & $-41,40$ & $-31,70$ & 0,1370 & 0,1050 \\
\hline
\end{tabular}

Tabela 10. Relações entre $\Delta \mathrm{G}_{\text {mic }}$ ou ads com o comprimento da cadeia alquílica $\left(\mathrm{NCH}_{2}\right) ; \Delta \mathrm{P}_{\text {ads }}^{\mathrm{o}}=\Delta \mathrm{P}_{\text {grupo cabeça }}^{\mathrm{o}}+\Delta \mathrm{P}_{\mathrm{CH} 3}^{\mathrm{o}}+\mathrm{NCH}_{2} \Delta \mathrm{P}_{\mathrm{CH} 2}^{\mathrm{o}}{ }^{*} \cdot \mathrm{APG}_{\mathrm{y}} \mathrm{C}_{\mathrm{y}}$, onde $\mathrm{y}=10,16$ ou 18

\begin{tabular}{|c|c|c|c|c|c|c|c|c|}
\hline \multirow{2}{*}{ Série } & \multicolumn{2}{|c|}{$\Delta \mathrm{G}_{\mathrm{ads}}^{\mathrm{o}}\left(\mathrm{kJ} \mathrm{mol}^{-1}\right)$} & \multicolumn{2}{|c|}{$\Delta \mathrm{G}_{\text {mic }}^{\mathrm{o}}\left(\mathrm{kJ} \mathrm{mol}^{-1}\right)$} & \multicolumn{2}{|c|}{$\Delta \mathrm{S}_{\text {ads }}^{\mathrm{o}}\left(\mathrm{kJ} \mathrm{mol}^{-1} \mathrm{~K}^{-1}\right)$} & \multicolumn{2}{|c|}{$\Delta \mathrm{S}_{\text {mic }}^{\mathrm{o}}\left(\mathrm{kJ} \mathrm{mol}^{-1} \mathrm{~K}^{-1}\right)$} \\
\hline & $\mathrm{A}^{\mathrm{a}}$ & $\mathrm{B}^{\mathrm{b}}$ & $\mathrm{A}^{\prime}$ & $\mathrm{B}^{\prime}$ & $A^{\prime}$ & $B^{\prime} \times 10^{-5}$ & $A^{\prime}$ & $B^{\prime} \times 10^{-4}$ \\
\hline $\mathrm{APG}-\mathrm{C}_{\mathrm{y}}$ & $-41,0$ & $-0,02$ & $-28,8$ & $-0,16$ & 0,14 & 9,6 & 0,09 & 6,0 \\
\hline
\end{tabular}

$* \mathrm{P}=$ parâmetro termodinâmico $(\mathrm{G}$ ou $\mathrm{S}),{ }^{\mathrm{a}} \mathrm{A}^{\prime}=\Delta \mathrm{P}_{\text {ads }}^{\mathrm{o}}=\Delta \mathrm{P}_{\text {grupo cabeça }}^{\mathrm{o}}+\Delta \mathrm{P}_{\mathrm{CH} 3}^{\mathrm{o}},{ }^{\mathrm{b}} \mathrm{B}^{\prime}=\Delta \mathrm{P}_{\mathrm{CH} 2}^{\mathrm{o}}$. 
Tabela 11. Valores de BHL para os alquil glicosídeos sintetizados e suas respectivas possíveis aplicações

\begin{tabular}{ccccc}
\hline Surfactantes & $\mathrm{M}_{\mathrm{h}}$ & $\mathrm{M}$ & BHL & Aplicações \\
\hline APG- $\mathrm{C}_{10}$ & 141 & 955,0 & 17,1 & Detergente \\
APG- $\mathrm{C}_{16}$ & 225 & 1039,0 & 15,7 & Emulsificante de óleo em água \\
APG- $\mathrm{C}_{18}$ & 253 & 1067,0 & 15,3 & Emulsificante de óleo em água \\
\hline
\end{tabular}

Obs: $\mathrm{M}_{\mathrm{h}}=$ massa molar da parte hidrofóbica e $\mathrm{M}=$ massa molar do surfactante.

a correspondente quantidade para a micelização $\left(-28,0 \mathrm{~kJ} \mathrm{~mol}^{-1}\right)$, como esperado para surfactantes com grupos hidrofílicos relativamente grandes. ${ }^{67}$

\section{Balanço Hidrofílico-Lipofílico (BHL)}

$\mathrm{O}$ valor de BHL mostra as possibilidades de aplicações para os alquil glicosídeos. Surfactantes com valor de BHL entre 9 e 16 são aplicados como emulsificantes de óleo em água, já os que possuem valores entre 16 e 19 são usados como detergentes. ${ }^{68}$ A Tabela 11 apresenta os valores de BHL calculados a partir da equação (19) e as aplicações para os surfactantes preparados neste trabalho.

Para os surfactantes sintetizados observou-se uma dependência linear entre $\mathrm{ln}$ cmc e BHL e BHL e $\Delta \mathrm{G}^{\circ}$ mic, de acordo com as relações encontradas por Lin e Marszall baseada na teoria de Davies, que relaciona a dependência do BHL com a contribuição dos grupos hidrofílicos e hidrofóbicos da molécula surfactante ${ }^{69-70}$ (Figura 23S $(\mathrm{a}, \mathrm{b})$ ). A relação linear entre BHL e $\Delta \mathrm{G}^{\mathrm{o}}$ mic associa o conceito de BHL com a tendência do surfactante para agregar ou adsorver na interface.

\section{CONCLUSÃO}

O método de Köenig-Knorr foi eficiente para a síntese dos APGs a partir da amilose. Os APGs obtidos são formados com cinco anéis de glicose por cadeia alquílica e a ligação dos álcoois à parte hidrofílica foi predominantemente $\beta$-glicosídica de acordo com as caracterizações de RMN ${ }^{1} \mathrm{H}, \mathrm{RMN}{ }^{13} \mathrm{C}$ e GPC. As análises térmicas (curvas de TGA e DSC) permitem constatar que o triacetato de amilose é mais estável termicamente e libera menos água durante a etapa inicial de decomposição do que a amilose, isso se dá pela entrada dos grupos acetilas que aumentam a sua lipofilicidade e promove a fusão em uma temperatura mais alta. Tanto a estabilidade térmica, quanto as temperaturas de fusão dos APGs crescem à medida que a cadeia alquílica é ampliada, devido ao aumento das interações de van der Waals nos cristais. Os surfactantes sintetizados apresentaram características semelhantes a outros surfactantes APGs, tais como cristais líquidos termotrópicos e liotrópicos, dado o aparecimento de pontos de fusão duplos nas curvas DSC. Verificou-se que a eficiência e a efetividade dos surfactantes de reduzirem a tensão superficial da água crescem com o aumento da cadeia alquílica. Entretanto, o contrário ocorre com os valores de tensão superficial na cmc e o cmc dos surfactantes sintetizados que diminuem com o aumento da cadeia alquílica, devido ao efeito hidrofóbico crescente. Comportamento similar ocorre com o tamanho da área por molécula (A), que pode ser justificado pelo empacotamento mais efetivo das moléculas dos surfactantes promovido por mais interações de van der Waals quando o tamanho da cadeia alquílica cresce. Valores de PCE mostram que os tipos de estrutura esperada para os agregados formados pelos surfactantes são de micelas cilíndricas relativamente grandes ou em formato de bastão e o tipo de emulsão esperada para os mesmos é do tipo óleo em água $(\mathrm{O} / \mathrm{A})$. Através dos parâmetros termodinâmicos verificou-se que as energias livres de Gibbs para os processos de adsorção são mais negativos do que aquelas para micelização e que em ambos os processos esta energia diminui com o aumento da cadeia alquílica, o que demonstra que os processos se tornam mais espontâneos à medida que aumenta a cadeia. Enfim, verificamos através dos valores de BHL que o APG- $\mathrm{C}_{10}$ pode ser aplicado como detergente e os APG- $\mathrm{C}_{16} \mathrm{e}$ $\mathrm{APG}-\mathrm{C}_{18}$ como emulsificantes de óleo em água. Este fato induz a estudos complementares para avaliação de outras propriedades que possam torná-los competitivos no mercado.

\section{MATERIAL SUPLEMENTAR}

Algumas imagens dos sistemas utilizados neste trabalho e detalhes da síntese dos alquil poliglicosídeos derivados da amilose estão disponíveis em http://quimicanova.sbq.org.br, na forma de arquivo PDF, com acesso livre.

\section{AGRADECIMENTOS}

CNPq (NMPSR-304392/2013-8), CAPES, CENAUREMN, INCT-NanoBioSimes e Central Analítica UFC/SISNANO.

\section{REFERÊNCIAS}

1. Lee, S.; Lee, J.; Yu, H.; Lim, J.; J. Ind. Eng. Chem. 2016, 38, 157.

2. Rybinski, W. V.; Hill, K.; Angew. Chem., Int. Ed. 1998, 37, 1328.

3. Messinger, H.; Aulmann, W.; Kleber, M.; Koehl, W.; Food Chem. Toxicol. 2007, 45, 1375.

4. Valente, A. J. M.; Nilsson, M.; Söderman, O.; J. Colloid Interface Sci. 2005, 281, 218

5. Lopes, C. A.; Silva, G. O.; Cruz, E. M.; Assad, E.; Pereira, A. S.; Hortic. Bras. 2011, 29, 7.

6. Ramos, V. J.; Watanabe, E. Y.; Ramos Junior, E. U.; Ito, M. A.; Oliveira, S. R.; Camargo, J. C. M.; Marchesin, M.; Factor, T. H.; Lima Junior, S.; Hortic. Bras. 2009, 27, S2708.

7. Grumezescu, A.; Food Packaging: Nanotechnology in the Agri-Food Industry, Vol. 7, Academic Press: New York, 2016.

8. Singh, N.; Singh, J.; Kaur, L.; Sodhi, N. S.; Gill, B. S.; Food Chem. 2003, 81, 219.

9. de França, F. C. F.; Coelho, E. de L.; Uchôa, A. F. J.; Rodrigues, F. H. A.; Ribeiro, M. E. N. P.; Soares, S. de A.; Ricardo, N. M. P. S.; Quim. Nova. 2016, 39, 771 .

10. Hill, K.; Rybinski, W. V.; Stoll, G.; Alkyl Polyglycosides: Technology, Properties and Applications, VCH Publishers Inc: New York, 1996.

11. Fité, F. X. C.; Text. Res. J. 2015, 85, 1455.

12. Charbonnier, V.; Morrison, B. M.; Paye, M.; Maibach, H. I.; Food Chem. Toxicol. 2001, 39, 279.

13. Effendy, I.; Maibach, H. I.; Contact Dermatitis 1995, 33, 217.

14. Sulek, M. W.; Wasilewski, T.; Wear 2006, 260, 193.

15. http://www.grandviewresearch.com/industry-analysis/alkylpolyglucosides-market, acessada em janeiro de 2017.

16. El-Sukkary, M. M. A.; Syed, N. A.; Aiad, I.; El-Azab, W. I. M.; J. Surfactants Deterg. 2008, 11, 129.

17. Ojha, S.; Mishra, S.; Kapoor, S.; Chand, S.; Appl. Microbiol. Biotechnol. 2013, 97, 5293

18. Marković, Z.; Predojevic, J.; Manojlovic, N. T.; Bull. Chem. Soc. Ethiop. 2011, 25, 83 
19. Milkereit, G.; Garamus, V. M.; Veermans, K.; Willumeit, R.; Vill, V.; J. Colloid Interface Sci. 2005, 284, 704.

20. Hughes, F. A.; Lew, B. W.; J. Am. Oil Chem. Soc. 1970, 47, 162.

21. Wurzburg, O. B. Em Methods in carbohydrates chemistry, v. 4, Whistler, R. L., ed.; Academic Press: New York, 1964, p. 288.

22. Bemiller, J.; Whistler, R.; Starch: Chemistry and Technology, Academic Press inc: New York, 2009.

23. Fringant, C.; Desbrières, J.; Rinaldo, M.; Polymers 1996, 37, 2663.

24. Miladinov, V. D.; Hanna, M. A.; Ind. Eng. Chem. Res. 1999, 38, 3892.

25. Mischnick, P.; Hennig, C.; Biomacromolecules 2001, 2, 180.

26. Ricardo, N. M. P. S.; Glendinning, A.; Price, C.; Polym. Eng. Sci. 1996, $36,182$.

27. Koenigs, W.; Knorr, E.; Chem. Ber. 1901, 34, 957.

28. Benoîte, L.; Chistophe, B.; Georges, M.; Jean, M. N.; Carbohydr. Res. 1997, 298, 251.

29. Mark, A. M.; Mehltretter C. L.; Starch 1972, 24, 73.

30. Colussi, R.; Halal, S. L. M. E.; Pinto, V. Z.; Bartz, J.; Gutkoski, L. C.; Zavareze, E. da R.; Dias, A. R. G.; LWT--Food Sci. Technol. 2015, 62, 1076.

31. Cornell, H. J.; Rix, C. J.; McGrane, S. J.; Starch/Stärke 2002, 54, 517.

32. Kitamura, S.; Kobayashi, K.; Tanahashi, H.; Ozaki, T.; Kuge, T.; J. Jpn. Soc. Starch Sci. 1989, 36, 257.

33. Brandrup, J.; Immergut, E. H.; Grulke, E. A.; Polymer Handbook, $4^{\mathrm{a}}$ ed., Wiley Interscience Publication: USA, 1999.

34. Coppola, L.; Gordano, A.; Procopio, A.; Sindona, G.; Colloids Surf., A 2002, 196, 175.

35. Jiang, L. C.; Basri, M.; Omar, D.; Rahman, M. B. A.; Salleh, A. B.; Rahman, R. N. Z. R. A.; J. Mol. Liq. 2011, 158, 175.

36. Israelachvili, J. N.; Colloids Surf., A 1994, 91, 1.

37. Binks, B. P.; Curr. Opin. Colloid Interface Sci. 2002, 7, 21.

38. Bazito, R. C.; Seould, O. A. E.; Langmuir 2002, 18, 4362.

39. Ferrer, M.; Comelles, F.; Plou, F. J.; Cruces, M. A.; Fuentes, G.; Parra, J. L.; Ballesteros, A.; Langmuir 2002, 18, 667.

40. Alam, M. S.; Kabir-ud-Din; Mandal, A. B.; J. Chem. Eng. Data 2010, $55,2630$.

41. Susan, M. A. B. H.; Ishibashi, A.; Takeoka, Y.; Watanabe, M.; Colloids and Surf., B 2004, 38, 167.

42. Bazito, R. C.; Seould, O. A. E.; Carbohydr. Res. 2001, 332, 95.

43. Bazito, R. C.; Seould, O. A. E.; J. Surfactants Deterg. 2001, 4, 395.

44. Maiti; Souvik; Chatterji, P. R.; J. Phys. Chem. B 2000, 104, 10253.
45. Hines, J. D.; Langmuir 2000, 16, 7575

46. Kelarakis, A.; Havredaki, V.; Rekatas, C. J.; Booth, C.; Phys. Chem. Chem. Phys. 2001, $3,1$.

47. Moroi, Y., Micelles: Theoretical and Applied Aspects, Plenum Press: New York, 1992.

48. Pavlovic, S.; Brandao, P. R. G.; Miner. Eng. 2003, 16, 1117.

49. Han, F.; Gao, C.; Liu, M.; Huang, F.; Zhang, B.; Int. J. Biol. Macromol. 2013, 59, 372.

50. Pascoal, A. M.; Maria, C. B.; Di-Medeiros; Batista, K. A.; Leles, M. I. G.; Lião, L. M.; Fernandes, K. F.; Carbohydr. Polym. 2013, 98, 1304.

51. http://www.thefreelibrary.com/New+Method+for+Surfactant+Quantifi cation+by+HPLC-GPC.-a072609459, acessada em maio 2016.

52. Wesslén, K. B.; Bengt, W.; Carbohydr. Polym. 2002, 47, 303

53. Hurtta, M.; Pitkänen, I.; Knuutinen, J.; Carbohydr. Res. 2004, 339, 2267.

54. Pielichowski, K.; Tomasik, P.; Sikora, M.; Carbohydr. Polym. 1998, 35, 49.

55. Miethchen, R.; Nols, J.; Prade, N.; Tetrahedron 1992, 48, 3061.

56. Minden, H. M. V.; Brandenburg, K.; Seydel, U.; Koch, M. H. J.; Garamus, V.; Willumeit, R.; Vill, V.; Chem. Phys. Lipids 2000, 106, 157.

57. Aggardwal, P.; Dollimore, D.; Thermochim. Acta 1998, 319, 17.

58. Aggardwal, P.; Dollimore, D.; Thermochim. Acta 1997, 291, 65.

59. Aggardwal, P.; Dollimore, D.; Thermochim. Acta 1998, 324, 1.

60. Kahl, H.; Enders, S.; Quitzsch, K.; Colloids Surf., A 2001, 183, 661.

61. Singh, V.; Isobe, S.; Toyoshima, H.; Okadome, H.; Ohtsubo, K.; Trends Appl. Sci. Res. 2007, 2, 175.

62. Szumata, P.; Mówinska, A.; J. Surfactants Deterg. 2016, 19, 437.

63. Rosevear, P.; Aken, T. V.; Baxter, J.; Ferguson-Miller, S.; Biochemistry 1980, 19, 4108 .

64. Ferrer, M.; Comelles, F.; Plou, F. J.; Cruces, M. A.; Fuentes, G.; Parra, J. L.; Ballesteros, A.; Langmuir 2001, 18, 667.

65. Israelachvili, J. N.; Mitchell, D. J.; Ninham, B. W.; J. Chem. Soc., Faraday Trans. 2 1976, 72, 1525.

66. Söderberg, I.; Drummond, C. J.; Furiong, D. N., Godkin, S.; Matthews, B.; Colloids Surf. A, 1995, 102, 91.

67. Rosen, M. J.; Surfactants and Interfacial Phenomena, Wiley: New York, 1989.

68. Materna, K.; Szymanowski, J.; J. Colloid Interface Sci. 2002, 255, 195.

69. Marszall, L.; Cosmet. Toiletries 1979, 94, 29.

70. Marszall, L.; Int. J. Pharm. 1980, 6, 253. 